\title{
Time-Varying MIMO Channels: Measurement, Analysis, and Modeling
}

Michael A. Jensen

jensen@byu.edu

Jon W. Wallace

wall@ieee.org

Follow this and additional works at: https://scholarsarchive.byu.edu/facpub

Part of the Electrical and Computer Engineering Commons

\section{Original Publication Citation}

Wallace, J. W., and M. A. Jensen. "Time-Varying MIMO Channels: Measurement, Analysis, and Modeling." Antennas and Propagation, IEEE Transactions on 54.11 (26): 3265-73

\section{BYU ScholarsArchive Citation}

Jensen, Michael A. and Wallace, Jon W., "Time-Varying MIMO Channels: Measurement, Analysis, and Modeling" (2006). Faculty Publications. 283.

https://scholarsarchive.byu.edu/facpub/283

This Peer-Reviewed Article is brought to you for free and open access by BYU ScholarsArchive. It has been accepted for inclusion in Faculty Publications by an authorized administrator of BYU ScholarsArchive. For more information, please contact ellen_amatangelo@byu.edu. 


\title{
Time-Varying MIMO Channels: Measurement, Analysis, and Modeling
}

\author{
Jon W. Wallace, Member, IEEE, and Michael A. Jensen, Senior Member, IEEE
}

\begin{abstract}
The temporal variation in measured multiple-input multiple-output (MIMO) wireless channels with moving communication nodes is analyzed. A wide-band $8 \times 8$ sounder is employed to measure the response of indoor and outdoor channels at 2.55 and $5.2 \mathrm{GHz}$. The rate of channel temporal variation is then quantified in terms of information theoretic metrics that indicate the loss in channel quality as transmit and receive channel state information becomes increasingly outdated. Finally, these metrics are used to investigate the ability of two different modeling strategies-a random matrix model and a physical time-varying cluster model-to capture the channel temporal variation.
\end{abstract}

Index Terms-Information theory, multiple-input multipleoutput (MIMO) systems, time-varying channels.

\section{INTRODUCTION}

$\mathbf{T}$ HE performance benefits of using multiple transmit and receive antennas for communicating over multipath fading channels have been well documented in the literature [1]. However, the effectiveness of this multiple-input multiple-output (MIMO) technology is highly dependent on the type and quality of channel state information (CSI) at the receiver and, in the case of rank-deficient channels, the transmitter [2]. In practice, CSI is obtained by dedicating a small fraction of the transmission bandwidth to known pilot symbols from which the receiver may estimate the channel transfer matrix. If the channel varies slowly relative to the symbol period (low node mobility and slow-moving scatterers), this training can be done infrequently with minimal impact on channel throughput. For highly mobile systems, however, the need for rapid training quickly exhausts the available transmission bandwidth, leading to low effective channel capacities.

While these observations suggest that accurate MIMO performance prediction requires a good representation of the channel temporal variation, most previous studies have focused only on the channel spatial properties. There have been a few papers on modeling channel temporal behavior [3], [4] or quantifying its impact on system performance [5], [6], although to date there has not been a complete and practical framework for evaluating

Manuscript received September 21, 2005; revised April 21, 2006. This work was supported in part by the National Science Foundation under Information Technology under Grants CCR-0313056 and CCF-0428004 and in part by the U.S. Army Research Office under Multi-University Research Initiative under Grant W911NF-04-1-0224.

J. W. Wallace was with the Department of Electrical and Computer Engineering, Brigham Young University, Provo, UT 84602 USA. He is currently with the School of Engineering and Science, International University of Bremen, Bremen D-28725, Germany (e-mail: wall@ieee.org).

M. A. Jensen is with the Department of Electrical and Computer Engineering, Brigham Young University, Provo, UT 84602 USA (e-mail: jensen@ee.byu. edu).

Digital Object Identifier 10.1109/TAP.2006.883985

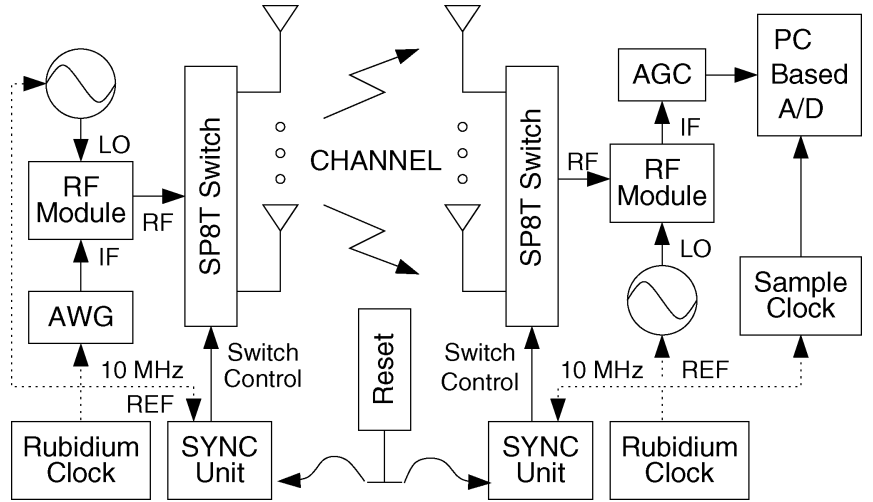

Fig. 1. Block diagram of the $8 \times 8$ wide-band MIMO channel sounder used to measure time variation of indoor and outdoor MIMO channels.

this variation. The purpose of this paper is to provide such a framework using observations obtained from applying quantitative metrics to indoor and outdoor MIMO channel measurements at 2.55 and $5.2 \mathrm{GHz}$. This paper also describes two different modeling strategies for time-variant MIMO channels-a multivariate complex normal (MVCN) model and a time-variant cluster (TVC) model - and compares their accuracy relative to the measured data.

\section{Channel Measurements}

Although channel modeling capabilities continue to improve [7], direct channel measurement still remains the only method for validating modeling results. In this paper, therefore, measurements in indoor and outdoor environments are used to develop suitable metrics for MIMO channel time-variation and to assess the accuracy of conventional modeling strategies.

\section{A. Measurement System}

Since this paper emphasizes the analysis of time-variant MIMO channels, we only briefly describe the measurement system and refer the reader to prior publications for additional details [8]-[10]. Fig. 1 depicts a block diagram of the wide-band $8 \times 8$ MIMO channel sounder used for this work. At the transmit side, an arbitrary waveform generator creates a multitone signal with up to $100 \mathrm{MHz}$ of instantaneous bandwidth which is up-converted to a radio-frequency carrier in the range of 2-8 GHz, amplified and fed to an eight-way microwave switch connected to the eight-element uniform circular array (UCA) of monopoles $(\lambda / 2$ interelement spacing where $\lambda$ is the wavelength). Each transmit element has its own power amplifier to avoid transmit power limitations imposed by the switch specifications. 


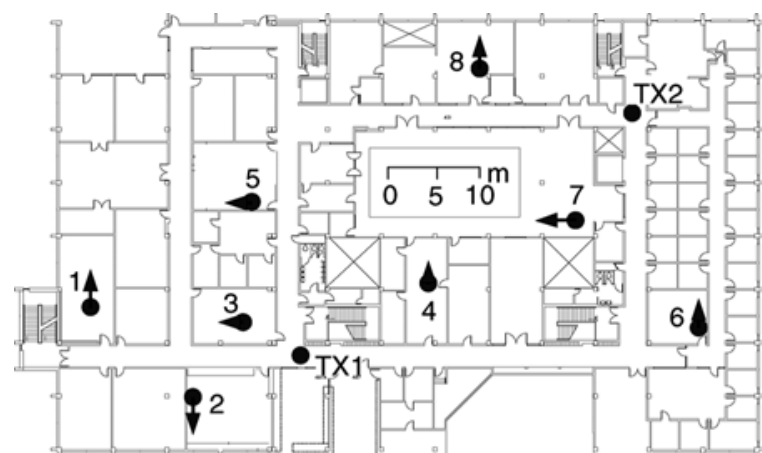

Fig. 2. Indoor measurement scenario, where $n$ indicates the $n$th receive location. TX 1 and 2 are the transmit locations for receive locations $1-5$ and 6-8, respectively. Circles and arrows indicate starting RX position and distance/direction moved, respectively.

At the receive side, an eight-way switch routes signals from the eight-element monopole UCA to a receiver that performs low noise amplification, down-conversion, automatic gain control (AGC), and analog-to-digital conversion on a personal computer (PC). Microwave switch control is performed by a customdesigned synchronization unit that scans all possible antenna combinations, where the number of antennas and dwell time are selectable. Highly stable $10 \mathrm{MHz}$ rubidium time/frequency references provide transmit/receive system synchronization. The receive waveforms and AGC levels are stored on the PC for channel coefficient estimation.

\section{B. Indoor Measurements}

The transmit signal used indoors consisted of eight tones with $10 \mathrm{MHz}$ separation (to ensure statistical independence) at a center frequency of either 2.55 or $5.2 \mathrm{GHz}$ and an average transmit power of $200 \mathrm{~mW}$. Channels were acquired with a $50 \mu$ s antenna dwell time either every $3.2 \mathrm{~ms}$ (fast mode, $1.875 \mathrm{~s}$ acquisition length) or every $25.6 \mathrm{~ms}$ (slow mode, $15 \mathrm{~s}$ acquisition length).

Fig. 2 depicts a floor plan of the indoor environment. The transmitter was stationary at two hallway positions, while the receiver was placed in eight different rooms and was moved at $30 \mathrm{~cm} / \mathrm{s}$ along the paths indicated. The cart speed was regulated by coordinating the cart position relative to marks on the floor and timing "beeps" generated by the computer, with a resulting speed error of approximately $\pm 1 \mathrm{~cm} / \mathrm{s}$.

In each room, an acquisition with the transmitter off and receiver stationary revealed that the level of cochannel interference was negligible. Next, measurements were performed with the transmitter on and receiver either stationary or moving (fast acquisition mode). Fig. 3 plots the resulting Doppler spectrum averaged over all eight indoor locations, with the $1 \mathrm{~Hz}$ maximum Doppler for stationary measurements being small compared to the 5-10 Hz maximum Doppler for moving measurements. These results reveal that the effects of moving scatterers in the environment are relatively unimportant and that even slow acquisition meets the Nyquist criterion for capturing the channel time variation.

\section{Outdoor Measurements}

The transmit signal used outdoors consisted of eight tones with $1 \mathrm{MHz}$ separation at a $2.55 \mathrm{GHz}$ center frequency and an

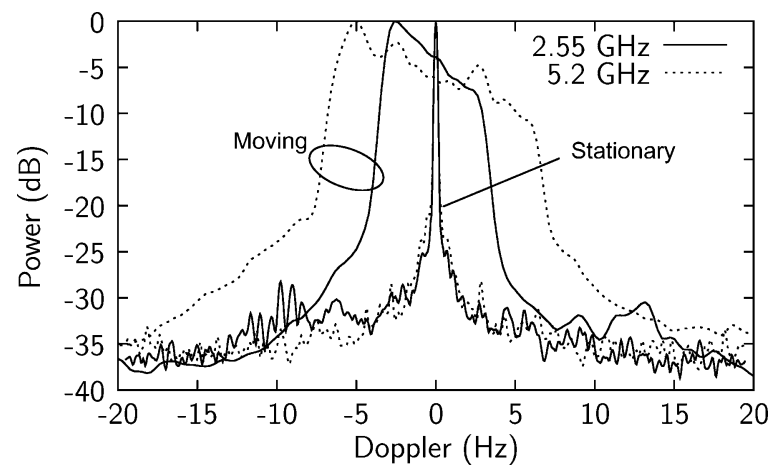

Fig. 3. Average Doppler spectrum for indoor measurements for locations 1-8 for stationary and moving measurements at 2.55 and $5.2 \mathrm{GHz}$.

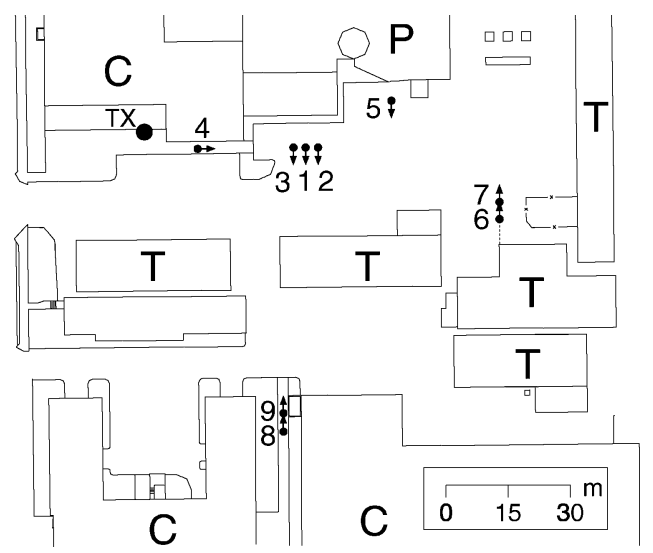

Fig. 4. Outdoor measurement scenario consisting of classroom buildings (C), temporary metal structures $(\mathrm{T})$, and a power plant $(\mathrm{P})$, where $n$ indicates the $n$th receive location and TX the transmit location. Circles and arrows indicate starting RX position and distance/direction moved, respectively.

average transmit power of $2 \mathrm{~W}$. Lack of suitable power amplifiers precluded outdoor measurements in the upper band. Because of the lower transmission bandwidth, fast $3.2 \mathrm{~ms}$ acquisition could be performed for a complete $15 \mathrm{~s}$.

Fig. 4 provides a map of the non-line-of-sight (NLOS) outdoor scenario consisting of a few classroom buildings (C) with brick and cinder block construction, several temporary $(\mathrm{T})$ metal structures, and a power production plant $(\mathrm{P})$. Parked cars flank the road on both sides of the street, and occasional moving cars are present. The transmitter was placed at a single position while the receiver was placed at nine different positions on the street or sidewalks. Fig. 5 depicts the average Doppler spectrum for measurements with the receiver stationary or moving at $30 \mathrm{~cm} / \mathrm{s}$, with respective maximum Dopplers of about 1 and $5 \mathrm{~Hz}$. Here again, the effect of moving scatterers appears to be small.

\section{MIMO TIME-VARIATION METRICS}

Key to this work is the definition of metrics that indicate the information-theoretic loss of channel quality as the channel varies. Although these metrics are intended for time-varying channels, we characterize variation versus movement distance, allowing the results to be scaled according to speed.

Consider the narrow-band MIMO system described by

$$
\mathbf{y}=\mathbf{H x}+\boldsymbol{\eta}
$$




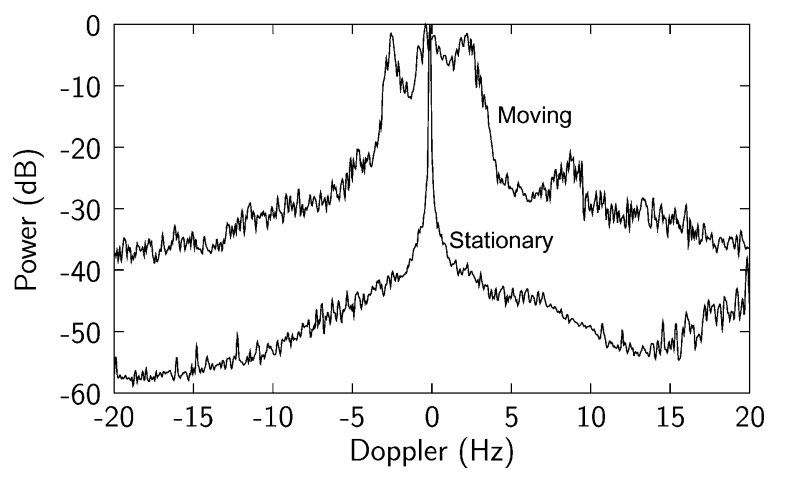

Fig. 5. Average Doppler spectrum for outdoor measurements for locations 1-9 for stationary and moving measurements at $2.55 \mathrm{GHz}$.

where $\mathbf{x}$ is the vector of input signals, $\mathbf{H}$ is the channel transfer matrix, $\boldsymbol{\eta}$ is the vector of receiver noise samples, and $\mathbf{y}$ is the vector of receive signals. In practice, the system uses a training sequence to obtain an initial estimate $\hat{\mathbf{H}}$ of the channel transfer matrix. Since this work emphasizes the channel evolution, we will consider this initial estimate to be error free.

We define capacity as a function of displacement $d$ as the instantaneous mutual information given knowledge of the channel at $d=0$. As $d$ increases, we expect a capacity reduction as the CSI becomes increasingly outdated. Such a capacity degradation metric is obviously linked to capacity under imperfect CSI where the initial channel estimate $\hat{\mathbf{H}}$ is in error [11]-[13]. The unique goal of this work, however, is to infer from MIMO measurements the elapsed time (or distance moved) beyond which an initial channel estimate is no longer suitable for high-capacity communications, information that is useful to the system designer who must choose transmit/receive CSI update rates and system adaptation strategies. We also point out that capacity degradation with outdated receive CSI has been studied by other researchers resulting in the development of similar metrics [5], [6], [14].

\section{A. Transmit CSI Delay (TCD)}

First, consider the case of transmit CSI delay (TCD) where the receiver has perfect CSI $(\mathbf{H})$ but the transmitter only has the delayed channel estimate $(\hat{\mathbf{H}})$. We define capacity for delayed transmit CSI as

$$
C_{T}(\mathbf{H}, \hat{\mathbf{H}})=\log _{2}\left|\frac{\mathbf{H Q}(\hat{\mathbf{H}}) \mathbf{H}^{H}}{\sigma^{2}}+\mathbf{I}\right|
$$

where $\mathbf{H}$ is the true channel, $\sigma^{2}$ is the receiver noise variance, $\mathbf{Q}(\hat{\mathbf{H}})$ is the optimal transmit covariance given by the waterfilling solution (assuming $\mathbf{H}=\hat{\mathbf{H}}$ ), $\mathbf{I}$ is the identity matrix, $\operatorname{Tr}\{\mathbf{Q}\} \leq P_{T}$, and $P_{T}$ is the total allowed transmit power. In this paper, $P_{T}$ and $\sigma^{2}$ are chosen so that the single-input singleoutput (SISO) signal-to-noise ratio averaged over all channel snapshots at a specific location is $10 \mathrm{~dB}$. As the estimate $\hat{\mathbf{H}}$ becomes increasingly outdated, $C_{T}$ will tend to decrease. The distance at which the TCD capacity drops below the uninformed transmit capacity $\left(C_{T}\right.$ with $\left.\mathrm{Q}=P_{T} / 8 \mathbf{I}\right)$ will be denoted as $d_{T}$.

This analysis is closely related to work in [15], where a connection is drawn between transmit correlation matrix distance (CMD), which is the distance over which the channel covariance remains essentially constant, and the bit error rate performance of a precoding scheme employing knowledge of the transmit covariance. We note that TCD quantifies the capacity loss independent of the type of modulation employed. Furthermore, $d_{T}$ provides a definite distance beyond which transmit CSI is no longer beneficial, whereas it is unclear how to derive such a critical distance from the CMD analysis.

\section{B. Receive CSI Delay (RCD)}

Next, consider the case of receive CSI delay (RCD), where both the transmitter and receiver have outdated CSI. If the transmitter and receiver attempt to form parallel Gaussian channels using the singular value decomposition of the delayed channel estimate $\left(\hat{\mathbf{H}}=\hat{\mathbf{U}} \hat{\mathbf{S}} \hat{\mathbf{V}}^{H}\right)$, we have

$$
\begin{aligned}
& \mathbf{y}=\hat{\mathbf{H}} \mathbf{x}+(\mathbf{H}-\hat{\mathbf{H}}) \mathbf{x}+\boldsymbol{\eta} \\
& \underbrace{\hat{\mathbf{U}}^{H} \mathbf{y}}_{\mathbf{y}^{\prime}}=\hat{\mathbf{S}} \underbrace{\left.\hat{\mathbf{V}}^{H} \mathbf{x}\right)}_{\mathbf{x}^{\prime}}+\underbrace{\hat{\mathbf{U}}^{H}(\mathbf{H}-\hat{\mathbf{H}}) \hat{\mathbf{V}}}_{\mathbf{M}}\left(\hat{\mathbf{V}}^{H} \mathbf{x}\right)+\underbrace{\hat{\mathbf{U}} \boldsymbol{\eta}}_{\boldsymbol{\eta}^{\prime}} .
\end{aligned}
$$

The approach constructs parallel channels with gains $\hat{S}_{i i}$ but creates self-interference controlled by the matrix $\mathbf{M}$.

For the RCD capacity to be completely general, no arbitrary constraints are placed on the time-variant behavior of $\mathbf{H}$, which leads to unknown statistics for $\mathbf{M}$. Therefore, the signaling strategy cannot be adapted to mitigate the effects of the self-interference. Since defining the capacity of this channel rigorously is difficult, we instead lower bound the capacity by computing the mutual information of a simplified system.

First, we assume that the transmitter uses the signaling strategy that is optimal for the case of no self-interference, or that $\mathbf{x}^{\prime}$ has zero-mean independent Gaussian elements with covariance $\mathbf{R}_{x}=\mathrm{E}\left\{\mathbf{x}^{\prime} \mathbf{x}^{\prime H}\right\}=\operatorname{diag}(\mathbf{p})$, where $p_{i}$ is the transmit power allocated to the $i$ th parallel channel. While the statistics of the self-interference term $\mathbf{z}=\mathbf{M x}^{\prime}$ are unknown, we make the analysis tractable and lower bound the capacity by assuming they are Gaussian. For a fixed transmit covariance $\mathbf{R}_{x}$, this leads to the mutual information

$$
C_{R}^{\prime}(\mathbf{H}, \hat{\mathbf{H}})=\log _{2}\left|\hat{\mathbf{H}} \mathbf{R}_{x} \hat{\mathbf{H}}^{H}\left(\mathbf{R}_{z}+\mathbf{I} \sigma^{2}\right)^{-1}+\mathbf{I}\right|
$$

where $\mathbf{R}_{z}=\mathrm{E}\left\{\mathbf{z z}^{H}\right\}$. Achieving this mutual information requires the receiver to know the covariance $\mathbf{R}_{z}$, which implies additional training. However, this contradicts our assumption that the receiver only has knowledge of $\hat{\mathbf{H}}$ at $d=0$.

A more realistic assumption is that the receiver knows the level of self-interference on the parallel subchannels but is unaware of the cross-correlation. Then $\mathbf{R}_{z}$ is assumed to be diagonal with entries $\left\{\mathbf{R}_{z}\right\}_{i i}=\left\{\mathbf{M} \mathbf{R}_{x} \mathbf{M}^{H}\right\}_{i i}$. For a fixed transmit covariance, the mutual information reduces to

$$
\begin{aligned}
C_{R}(\mathbf{H}, \hat{\mathbf{H}}) & =\sum_{i} \log _{2}\left(1+p_{i} \hat{S}_{i i}^{2} / q_{i}\right) \\
q_{i} & =\left\{\mathbf{M} \mathbf{R}_{x} \mathbf{M}^{H}\right\}_{i i}+\sigma^{2} \\
\mathbf{M} & =\hat{\mathbf{U}}^{H} \mathbf{H} \hat{\mathbf{V}}-\mathbf{\Phi} \hat{\mathbf{S}}
\end{aligned}
$$

where $p_{i}$ are found according to water-filling (assuming $\mathbf{H}=\hat{\mathbf{H}}$ and $\left.q_{i}=\sigma^{2}\right), \mathbf{p}=\operatorname{diag}\left(\mathbf{R}_{x}\right)$, and $\boldsymbol{\Phi}$ is a diagonal matrix with $\left|\Phi_{i i}\right|=1$. We define $d_{R}$ as the distance at which $C_{R}$ drops to $50 \%$ of its maximum value. 
When $\boldsymbol{\Phi}=\mathbf{I}$, the definitions for $\mathbf{M}$ in (4) and (8) are identical $\left(\hat{\mathbf{H}}=\hat{\mathbf{U}} \hat{\mathbf{S}} \hat{\mathbf{V}}^{H}\right)$, and $\mathbf{M}$ therefore includes the effect of changing phase on the parallel communication channels. However, channel phase variation is uninteresting from the standpoint of time-varying multipath structure since it can arise from system considerations (local oscillator drift, temperature variations), and its impact can be removed by using differential modulation. Therefore, setting $\arg \left(\Phi_{i i}\right)=\arg \left(\left\{\hat{\mathbf{U}}^{H} \mathbf{H} \hat{\mathbf{V}}\right\}_{i i}\right)$ will remove the impact of phase variation of the individual parallel channels and emphasize the impact of the changing channel spatial structure.

The mutual information expression in (6) does not represent actual capacity for a number of reasons. Obviously, the assumptions of independent Gaussian interference will not be true in general. Also, we have neglected the possibility that the transmitter and receiver work together to learn the statistical nature of the time-varying channel and adapt their signaling strategy to minimize the self-interference. Although we have investigated this possibility, in practice the capacity gains for such a strategy are usually only modest. Finally, the definition of capacity requires an infinite-time coding window over which channel statistics are stationary, which will clearly not be the case for the arbitrary fading channels we consider. Despite these shortcomings, we feel that the definition is useful as a numerical measure of the level of time variability of MIMO channels in an information theoretic sense.

\section{Averaging}

To allow observation of the average channel time-variation behavior, we compute average capacities as

$$
C_{T, R}(m d)=\frac{1}{M} \sum_{k=1}^{N_{F}} \sum_{n=1}^{N-m} C_{T, R}\left(\mathbf{H}^{(k, n+m)}, \mathbf{H}^{(k, n)}\right)
$$

where $\mathbf{H}^{(k, n)}$ is the channel transfer matrix for frequency bin $k$ and distance index $n, d$ is the separation distance of samples, $M=N_{F}(N-m)$, and $N_{F}$ and $N$ are the number of frequency bins and distance samples, respectively.

\section{Measured TCD and RCD Capacity}

In this section, we analyze the TCD and RCD capacity metrics for MIMO channels measured in the indoor and outdoor scenarios described in Section II.

1) Indoor Channels: Fig. 6 plots the TCD capacity metric $C_{T}$ normalized by the maximum value (zero displacement) as a function of distance for the two frequencies. The three sets of curves correspond to the average, maximum, and minimum values over all eight locations and all frequencies. The displacement is represented as physical distance (meters) as opposed to electrical distance (wavelengths) since this scale leads to high agreement between the curves at the two frequencies. This fact suggests that capacity loss due to delayed transmit CSI arises mainly from long-term changes in the multipath structure (direction and power) and not the small-scale fading. The distance $d_{T}$ at which the capacity curve intersects the uninformed transmit capacity $\left(C_{U T}\right)$ represents the distance beyond which transmit CSI is no longer useful.

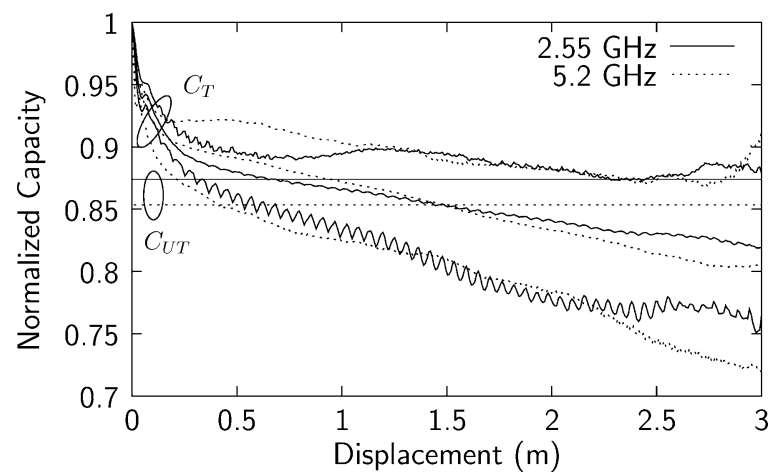

Fig. 6. Normalized capacity with delayed transmit CSI $\left(C_{T}\right)$ for indoor locations $1-8$, with curves for maximum, minimum, and mean values at each frequency. The average absolute capacities are 17.7 and $16.6 \mathrm{bits} / \mathrm{s} / \mathrm{Hz}$ at 2.55 and $5.2 \mathrm{GHz}$, respectively.

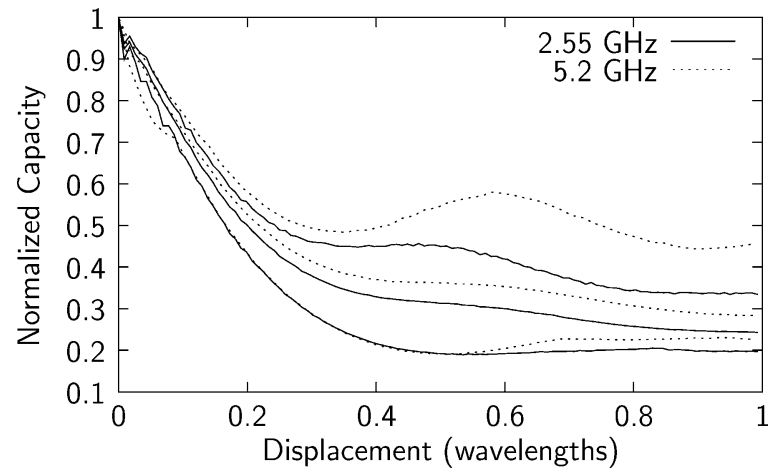

Fig. 7. Normalized capacity with delayed receive CSI for indoor locations 1-8, with curves for maximum, minimum, and mean values at each frequency.

It is noteworthy that $d_{T} \approx 0.6$ and $1.5 \mathrm{~m}$ for the 2.55 and 5.2 $\mathrm{GHz}$ measurements, respectively. These relatively large values suggest that even infrequent training can benefit performance, somewhat in contrast to conventional wisdom that transmit CSI is impractical due to the required feedback. This observation is similar to that made in [15], where transmit precoding was shown to be beneficial for CSI delays induced by movement of up to about $1.5 \mathrm{~m}$ at $2.45 \mathrm{GHz}$.

Fig. 7 plots the normalized RCD capacity as a function of electrical distance at the two frequencies. The similarity of the curves for the two frequencies versus electrical distance suggests that fading controls RCD capacity. The rapid decay in $C_{R}$ with distance $\left(d_{R} \approx \lambda / 4\right)$ indicates that training is required frequently (every $\lambda / 10$ ) to maintain high capacity.

The difference in distances over which the transmit and receive CSI is useful stems from the fact that the transmitter need only ensure that the signals are sent in proper directions for achieving good receiver signal quality. As this depends on the multipath spatial structure (angles/gains of departure), this typically varies slowly with distance. The receiver, however, must be able to invert the channel matrix to extract the parallel data streams, a task that requires accurate CSI.

Fig. 8 plots the mean capacity for indoor locations $1-8$ assuming knowledge of either all elements or the diagonal elements of $\mathbf{R}_{z}$, given by (5) and (6), respectively. TCD capacity (perfect knowledge of $\mathbf{M}$ at the receiver) is also plotted for comparison. The results indicate that about half of the capacity loss 


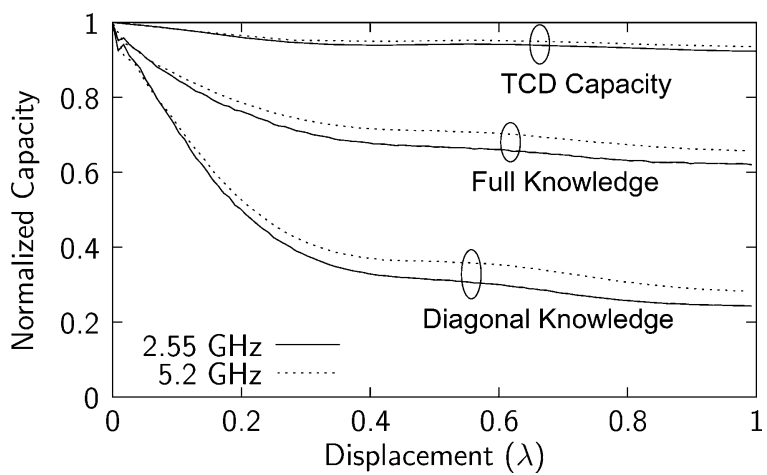

Fig. 8. Effect of the knowledge of the self-interference on capacity width delayed receive CSI for indoor locations $1-8$.

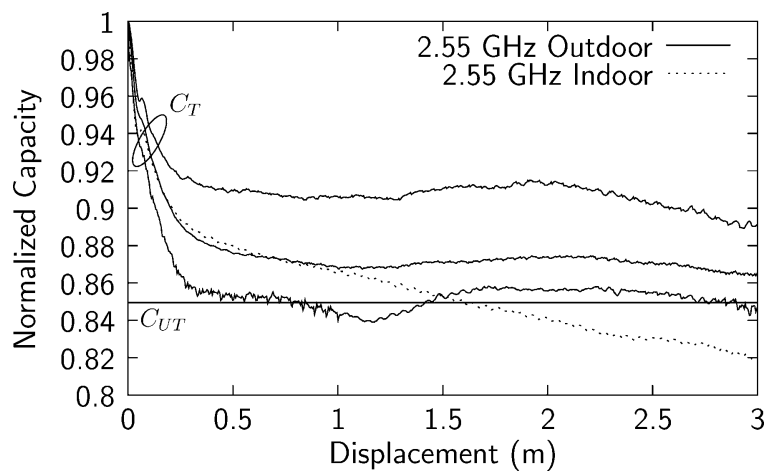

Fig. 9. Normalized capacity with delayed transmit CSI for outdoor locations $1-9$, with curves for maximum, minimum, and mean values at $2.55 \mathrm{GHz}$. Also plotted are the average uninformed transmit capacity for the outdoor cases and the mean TCD capacity for indoor locations.

arises from ignorance of the cross-correlation terms, implying that using additional training to track $\mathbf{R}_{z}$ could improve performance. If $\mathbf{R}_{z}$ is slowly varying, such tracking should require modest training requirements. However, this is beyond the scope of this paper, and the case of full knowledge of $\mathbf{R}_{z}$ will not be considered further.

2) Outdoor Channels: Fig. 9 illustrates the TCD metric for measured outdoor channels at $2.55 \mathrm{GHz}$, where again average, maximum, and minimum values are included. The average indoor TCD metric at $2.55 \mathrm{GHz}$ as well as the average outdoor uninformed transmit capacity are also shown. Comparison of the data reveals that the long-term decay of the outdoor TCD capacity is much slower than that of the indoor value, with $d_{T}>3 \mathrm{~m}$ on average.

Fig. 10 plots the RCD capacity as a function of electrical distance for outdoor locations 1-9 along with the average indoor RCD capacity. Interestingly, the outdoor $d_{R}$ value for the average curve is only slightly longer than that for the indoor measurements. This similarity is remarkable, since the two channels should have very different scattering characteristics, further suggesting that RCD capacity reduction is somewhat insensitive to the exact multipath structure.

\section{Time-VARiant MiMO Channel Models}

Although measured data provide the most accurate representation of channel time variation, the time-consuming expensive nature of data collection limits our ability to experimentally examine all possible channel configurations. In contrast, accurate

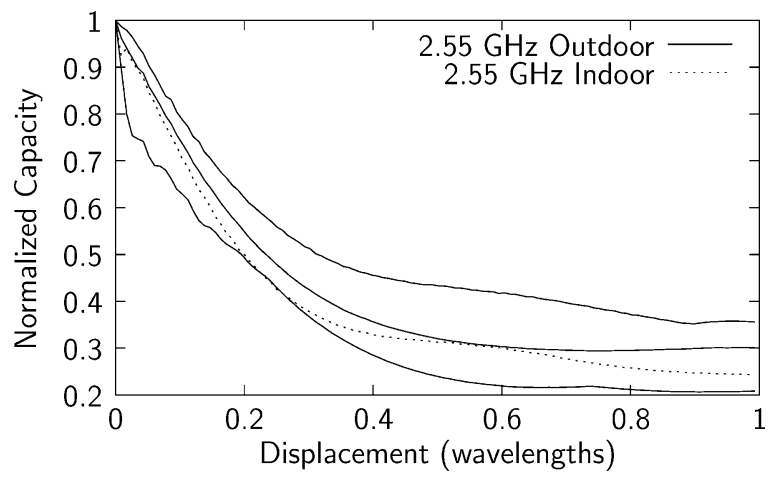

Fig. 10. Normalized capacity with delayed receive CSI for outdoor locations 1-9, with curves for maximum, minimum, and mean values at $2.55 \mathrm{GHz}$. Also plotted is the mean RCD capacity for indoor locations.

channel models are typically very convenient due to low cost and ease of simulation. Parameters for such models can be estimated from measured data or hypothesized based on realistic assumptions, allowing a designer to easily simulate a wide range of environments.

Our goal is to explore the extension of conventional MIMO channel modeling techniques to time-varying channels by extracting model parameters from the measured data and using the metrics in Section III to determine if the models capture the correct channel behavior. Although many models exist, we focus on 1) a random matrix model following the MVCN distribution and 2) a physical TVC model. The MVCN model parameters can be directly estimated from collected data but can be difficult to interpret physically. In contrast, the TVC model exhibits a compelling physical interpretation, although unique extraction of cluster parameters can be difficult.

\section{A. MVCN Model}

We represent the complex gain from the $j$ th transmitter to the $i$ th receiver at time index $n$ for a single frequency bin as $H_{i j}^{(n)}$. If these gains follow a (possibly time-dependent) MVCN distribution in both time and space, the spatiotemporal variation of the MIMO channel is completely characterized by the multivariate mean $(\boldsymbol{\mu})$ and covariance $(\mathbf{R})$, or

$$
\begin{aligned}
\mu_{i j}^{(n)} & =\mathrm{E}\left\{H_{i j}^{(n)}\right\} \\
R_{i j, k \ell}^{(n, m)} & =\mathrm{E}\left\{\left(H_{i j}^{(n)}-\mu_{i j}^{(n)}\right)\left(H_{k \ell}^{(n+m)}-\mu_{k \ell}^{(n+m)}\right)^{*}\right\}
\end{aligned}
$$

where $\mathrm{E}\{\cdot\}$ is expectation. For a stationary distribution, $\boldsymbol{\mu}$ and $\mathbf{R}$ are not a function of $n$ and can be obtained with sample averages. The difficulty of extracting these parameters from a nonstationary process depends on the severity of the nonstationarity, and may even be impossible for overspread processes [16]. Here, we consider a process characterized by a mean and covariance that vary slowly in time, allowing estimation by weighted sample averages or

$$
\begin{aligned}
\hat{\mu}_{i j}^{(n)} & =\sum_{s=-\infty}^{\infty} w_{s} H_{i j}^{(n+s)} \\
\hat{R}_{i j, k \ell}^{(n, m)} & =\sum_{s=-\infty}^{\infty} w_{s+m / 2} Z_{i j}^{(n+s)} Z_{k \ell}^{(n+s+m) *}
\end{aligned}
$$


where $Z_{i j}^{(n)}=H_{i j}^{(n)}-\hat{\mu}_{i j}^{(n)}$ and $w_{s}$ is the weighting window shifted in (13) to apply a weight of $w_{0}$ when the points $n+s$ and $n+s+m$ are equidistant from the estimation point $n$.

The choice of the window is a tradeoff between the bias and variance of the estimator, and optimal windows may be specified if prior information about the distribution is available [17]. In this paper, we apply an exponential window of the form $w_{s}=$ $\exp \left(-\left|s / \ell_{c}\right|\right)$, where $\ell_{c}$ is the correlation length. If the process is determined to be nearly stationary over $N_{s}$ samples, faithful estimates can be obtained with $4 \ell_{c}=N_{s}$.

1) Channel Stationarity: Determining the distance over which the channel can be considered a stationary process is nontrivial. One option is application of direct statistical tests for multivariate normality [18] applied to the channel data stacked into a vector. If either the data are nonnormal or moments are time-variant, the tests should fail. Thus, $N_{s}$ can be determined by increasing the size of the data window until these statistical tests begin to indicate nonconformance. Another option is to use the CMD measure [15], which focuses only on the change in sample covariance and avoids rejection due to data nonnormality. However, this approach suffers from the difficulties that 1) the data generating each sample covariance must represent a stationary process (which must somehow be determined) and 2) the threshold value of CMD to use for determining nonstationarity is unclear.

Because of the difficulties associated with application of CMD to the present problem, only the direct tests of multivariate normality are considered. Because no single test is robust against all possible alternative distributions, several tests should be applied to assess normality [19]. We determine a suitable value for $\ell_{c}$ by applying three different tests for multivariate normality: 1) Mardia's tests for multivariate skewness, 2) kurtosis [20], and 3) the Henze-Zirkler test [21] with $\beta=0.5$. One problem of applying these tests is that MIMO channels are apparently not strictly MVCN for large numbers of antennas [18]. Although this presents possible technical difficulties for the MVCN model, assuming MVCN statistics seems to be the only logical and practical starting point. Since we are mainly interested in testing the temporal stationarity of the process (whether constant first- and second-order statistics represent the data) and not the normality of the data, we apply the tests to all $2 \times 2$ antenna subsets of the data, rather than the full $8 \times 8$ data. Thus, the tests should indicate for what window size the data are temporally stationary, even if the full $8 \times 8$ spatial distributions are not MVCN.

Fig. 11 depicts the average rejection rates for a significance level of 5\% and a varying record length for indoor locations $1-8$ at $2.55 \mathrm{GHz}$. The results indicate that over distances of $4-8 \lambda$, the rejection rates are near the optimal $5 \%$, and we therefore let $\ell_{c}=2 \lambda / \Delta$, where $\Delta$ is the sample spacing $\left(4 \ell_{c}=N_{s}\right)$. Applying this test to the $5.2 \mathrm{GHz}$ indoor measurements leads to the same value of $\ell_{c}$.

For outdoor measurements, we found that frequency bins spaced by $1 \mathrm{MHz}$ were somewhat correlated for our environment, and therefore used only four frequency bins spaced by 2 $\mathrm{MHz}$ to improve the independence of the frequency samples. The results indicate that the tests give rejection rates acceptably close to $5 \%$ for a record length of $8-16 \lambda$, implying $\ell_{c}=4 \lambda / \Delta$ for outdoor measurements.

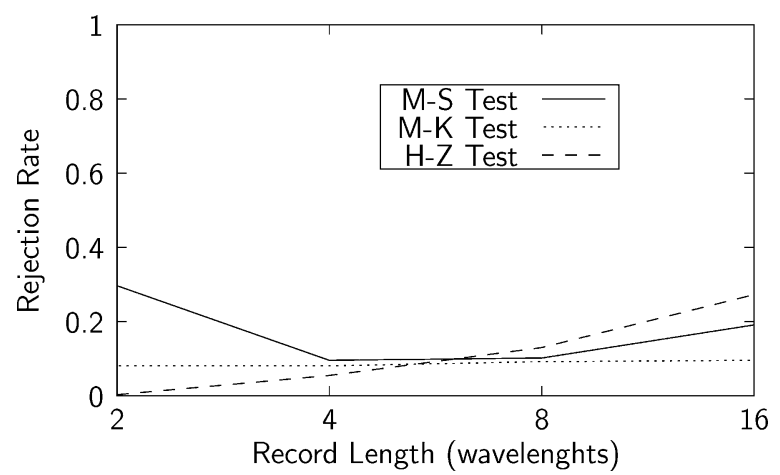

Fig. 11. Average rejection rates for three multivariate normal tests for indoor locations $1-8$ at $2.55 \mathrm{GHz}$.

2) Synthetic Channel Generation: Once the time-varying mean and covariance have been estimated from the data via (13), we require a way of generating simulated channels. This can be accomplished by forming the full space-time covariance matrix and using it to correlate the elements of i.i.d. complex normal vectors. This approach is numerically prohibitive, however, since for eight transmitters and receivers and 500 time steps, the covariance matrix has dimensions $32000 \times 32000$. Another approach involves modeling the channel as the output of an autoregressive filter, with weights obtained from the block Yule-Walker equations, fed by spatially and temporally white noise. In practice, however, large arrays and time windows lead to an ill-conditioned system which cannot be easily solved.

Perhaps the simplest approach is to assume that the covariance is separable in the time and space or $R_{i j, k \ell}^{(n, m)}=$ $R_{S, i j, k \ell}^{(n)} R_{T}^{(n, m)}$. Values for the separate space and time covariances are obtained by averaging the full covariance over all time steps and antennas, respectively. The synthetic channels are generated stepwise as

$$
\begin{aligned}
B_{i j}^{(n)} & =\sum_{n^{\prime}} X_{T, n n^{\prime}} A_{i j}^{\left(n^{\prime}\right)} \\
H_{i j}^{(n)} & =\sum_{i^{\prime} j^{\prime}} X_{S, i j, i^{\prime} j^{\prime}}^{(n)} B_{i^{\prime} j^{\prime}}^{(n)}
\end{aligned}
$$

where $\mathbf{X}_{T}=\mathbf{R}_{T}^{\prime 1 / 2}, \mathbf{X}_{S}^{(n)}=\mathbf{R}_{S}^{(n) 1 / 2}, R_{T, n n^{\prime}}^{\prime}=R_{T}^{\left(n, n^{\prime}-n\right)}, i$ and $j$ are stacked when used as a covariance index, and $A_{i j}^{(n)}$ are i.i.d. complex normal random variables.

To reduce the number of model parameters, an average value for the temporal correlation is used at each time step, or

$$
R_{T}^{(n, k)}=(1 / N) \sum_{n^{\prime}=1}^{N} \hat{R}_{T}^{\left(n^{\prime}, k\right)}
$$

where $N$ is the number of time steps considered and $\hat{\mathbf{R}}_{T}^{(n, m)}$ is the raw estimate of the temporal correlation from the collected data. We refer to this model with a coherent average of the temporal correlations as $\mathrm{MVCN}(\mathrm{CE})$, where $\mathrm{CE}$ stands for complex envelope. On the other hand, the averaging can be performed incoherently as

$$
R_{T}^{(n, k)}=(1 / N) \sum_{n^{\prime}=1}^{N}\left|\hat{R}_{T}^{\left(n^{\prime}, k\right)}\right|
$$

and this is referred to as $\mathrm{MVCN}(\mathrm{PE})$ for power envelope. 
Forcing this space-time separability and averaging the temporal correlation reduces the accuracy of the MVCN model. However, such simplifications seem necessary to arrive at a model that is reasonable in terms of both computational burden and parametric complexity.

\section{B. TVC Model}

The double-directional channel concept [22] is a powerful technique for system-independent representation of spatial channels, and much research effort has been dedicated to extracting the parameters for individual multipath components from measured data [23], [24]. Alternatively, we can treat the channel as an incoherent process described by a double-directional power spectrum [25]. This method groups multipath components into clusters of arrivals and departures and estimates only the cluster parameters.

1) Cluster Extraction: We first compute the double-directional Bartlett spectrum at time step $n$ from the data according to [25]

$$
P^{(n)}(\Omega)=\mathbf{b}^{H}(\Omega) \mathbf{R}_{S}^{(n)} \mathbf{b}(\Omega)
$$

where $\Omega=\left(\phi_{T}, \phi_{R}\right), b_{i k}(\Omega)=\psi_{R, i}\left(\phi_{R}\right) \psi_{T, k}\left(\phi_{T}\right)$ is the joint steering vector with $\psi_{S, i}\left(\phi_{S}\right)=\exp \left[j 2 \pi\left(x_{S, i} \cos \phi_{S}+\right.\right.$ $\left.y_{S, i} \sin \phi_{S}\right)$ ], $i$ and $k$ become a single stacked index, $S$ is either $T$ or $R$ for transmit or receive, $\phi_{S}$ is azimuth angle, and $x_{i}$ and $y_{i}$ are $x$ and $y$ coordinates of the $i$ th antenna. Given a true incoherent arrival power spectrum of $A(\Omega)$, the covariance is

$$
\mathbf{R}=\int d \Omega A(\Omega) \mathbf{\Psi}(\Omega)
$$

where $\boldsymbol{\Psi}(\Omega)=\boldsymbol{\Psi}_{T}\left(\phi_{T}\right) \otimes \boldsymbol{\Psi}_{R}\left(\phi_{R}\right)(\otimes$ is the Kronecker product) and $\Psi_{S}\left(\phi_{S}\right)=\psi_{S}\left(\phi_{S}\right) \psi_{S}\left(\phi_{S}\right)^{H}$. Decomposing the true spectrum into basis functions (clusters) $A_{p}(\Omega)$, we have

$$
A(\Omega)=\sum_{p} a_{p} A_{p}(\Omega)
$$

and the Bartlett spectrum becomes

$$
\begin{aligned}
P^{(n)}(\Omega) & =W[A]=\int d \Omega^{\prime} A\left(\Omega^{\prime}\right) \mathbf{b}^{H}(\Omega) \Psi\left(\Omega^{\prime}\right) \mathbf{b}(\Omega) \\
& =\sum_{p} a_{p} W\left[A_{p}\right] .
\end{aligned}
$$

The advantages of using the Bartlett spectrum instead of the covariance are: 1) the resulting equations are real and 2) covariance structure representing nonpropagating modes is removed. By discretizing all functions of $\Omega$ and matching left- and righthand sides at a number of discrete points, we obtain the matrix equation $\mathbf{p}=\mathbf{W a}$, which can be solved via linear programming for the positive real basis coefficients $a_{p}$. In practice, the linear-programming method returns a fairly sparse solution, consisting of only a small set of nonzero coefficients, which we refer to loosely as "clusters."

In this paper, we assume a set of Gaussian-shaped basis functions (clusters) with possible arrival angles of $\left\{0^{\circ}, 5^{\circ}, 10^{\circ}, \ldots, 355^{\circ}\right\}$ and angular spreads of $\left\{5^{\circ}, 10^{\circ}, 20^{\circ}, 40^{\circ}\right\}$. The time-variant nature of the clusters is obtained by the following steps.
1) The average Bartlett spectrum is computed for all time steps in a data record.

2) A set of clusters is estimated from the average Bartlett spectrum, and the dominant clusters, representing $90 \%$ of the channel power, are retained.

3) The time-variant Bartlett spectrum is computed for each time step.

4) Using linear programming, best fit values for $a_{p}$ are estimated at each time step for the reduced set of clusters found in step 2).

Since the full joint double-directional estimation problem results in a very large coefficient matrix $\mathbf{W}$, we simplify the method by first estimating one-dimensional clusters for single-directional transmit and receive to determine which basis functions are significant. Then, only the significant clusters are used in the joint two-dimensional estimation.

2) Synthetic Channel Generation: Synthetic channels are generated by assuming $L$ rays per cluster and computing the channel response as

$$
H_{i j}^{(n)}=L^{-1 / 2} \sum_{p, \ell} a_{p}^{(n) 1 / 2} \beta_{p \ell} \psi_{R, i}\left(\phi_{R, p \ell}\right) \psi_{T, j}\left(\phi_{T, p \ell}\right)
$$

where $\phi_{S, p \ell} \sim \mathcal{N}\left(\bar{\phi}_{S, p}, \sigma_{S, p}^{2}\right), \beta_{p \ell} \sim \mathcal{C N}(0,1), \bar{\phi}_{S, p}$ and $\sigma_{S, p}^{2}$ are the mean and variance of the departures/arrivals for cluster $p$, and $\mathcal{N}\left(\mu, \sigma^{2}\right)$ and $\mathcal{C N}\left(\mu, \sigma^{2}\right)$ are the real and complex normal distributions with mean $\mu$ and variance $\sigma^{2}$, respectively. Also, note that $\psi_{S}$ depends implicitly on $n$, since antenna position changes in time. Extensions to the model include allowing a different number of rays (richness) in each cluster, rays that dynamically appear or disappear in time, a time-variant set of clusters, etc. For this paper, a fixed set of $L=50$ rays per cluster is assumed for each realization of the model. Also, note that this current model only attempts to fit the channel covariance and has no provision for including nonfading components (channel mean).

\section{Example Application}

Fig. 12 plots an example of the average double-directional spectrum estimate for indoor location 6 . In nearly all cases, only a few $(<10)$ clusters are required to represent the specified $90 \%$ of the time-average power spectrum. Fig. 13 depicts the time variation of the cluster coefficients for location 6 for the three dominant clusters. Also shown is the amount of residual fractional error in the fit, which is close to the ideal 10\%. A common feature to nearly all indoor and outdoor locations is that the same set of clusters remains important over the complete $4.5 \mathrm{~m}$ path, although the overall power can change dramatically over this distance.

\section{Model Comparisons}

We now compare the results of applying the TCD and RCD capacity metrics to the models and measured data. We show only the results for indoor locations $1-8$ at $2.55 \mathrm{GHz}$, since all other results lead to similar conclusions.

Fig. 14 plots the fractional root mean square (rms) error in the RCD metric as a function of distance for indoor locations 1-8 for three different models. The discrepancy between the 


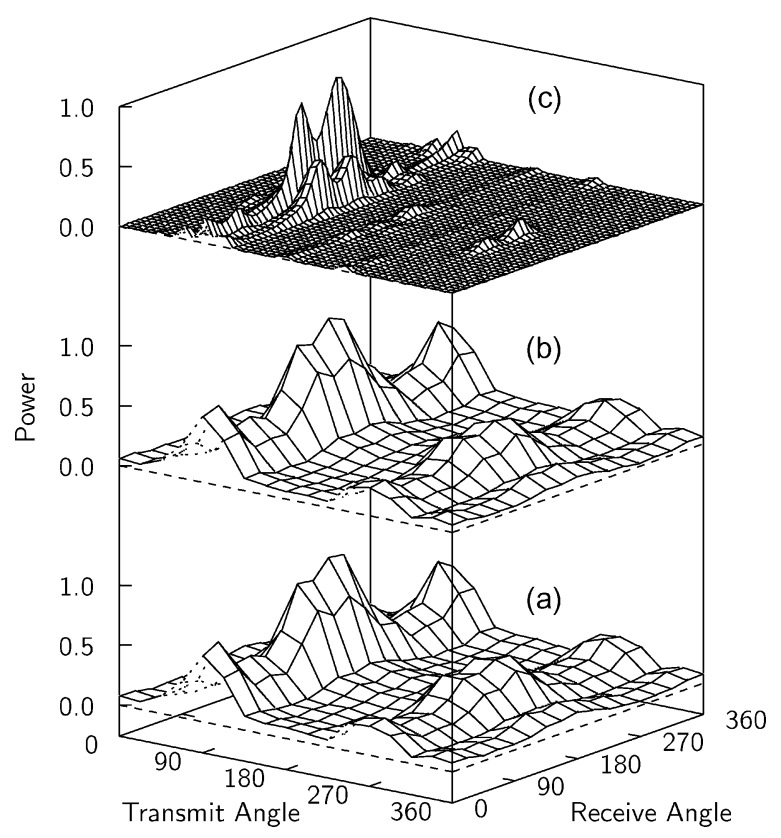

Fig. 12. Example time-average spatial spectrum estimate for indoor Location 6 at $2.55 \mathrm{GHz}$ : (a) measured and (b) modeled Bartlett spatial spectra and (c) estimated true spectrum.

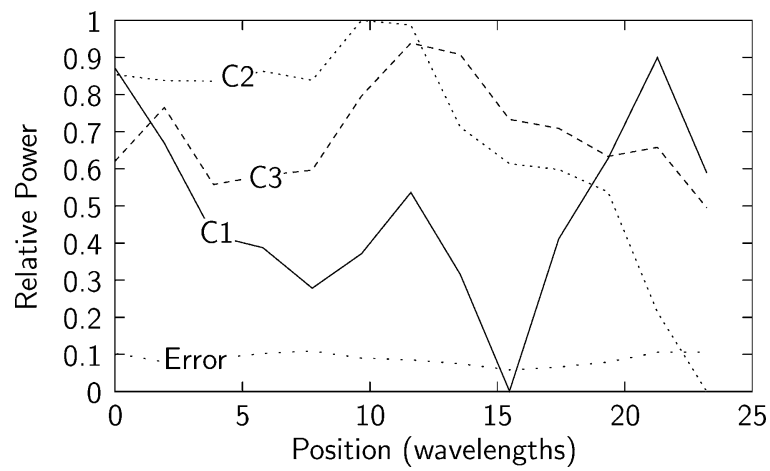

Fig. 13. Variation of the cluster power coefficients versus movement distance for indoor location 6 at $2.55 \mathrm{GHz}$ for the dominant three clusters $(\mathrm{C} 1-\mathrm{C} 3)$ as well as the fractional error of the fit.

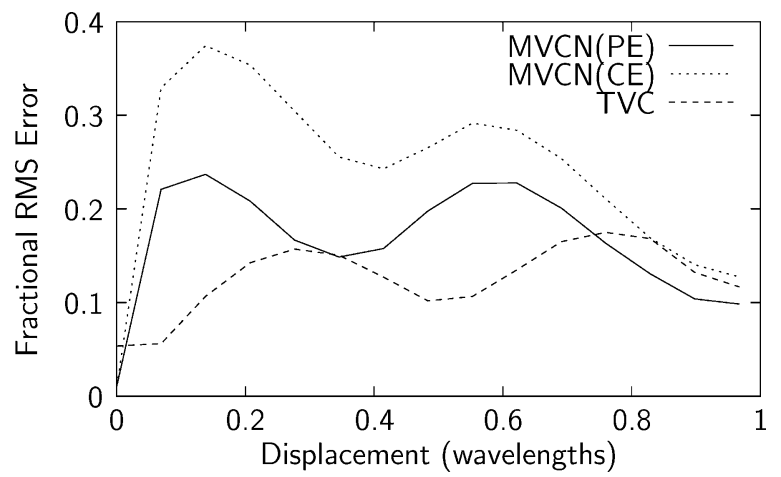

Fig. 14. RMS error in the RCD capacity metric for the TVC model and MVCN model with PE and complex envelope (CE) temporal correlation.

$\operatorname{MVCN}(\mathrm{CE})$ and $\mathrm{MVCN}(\mathrm{PE})$ results may stem from the fact that the coherent averaging in (16) will tend to underestimate the temporal correlation if the process is not stationary over the

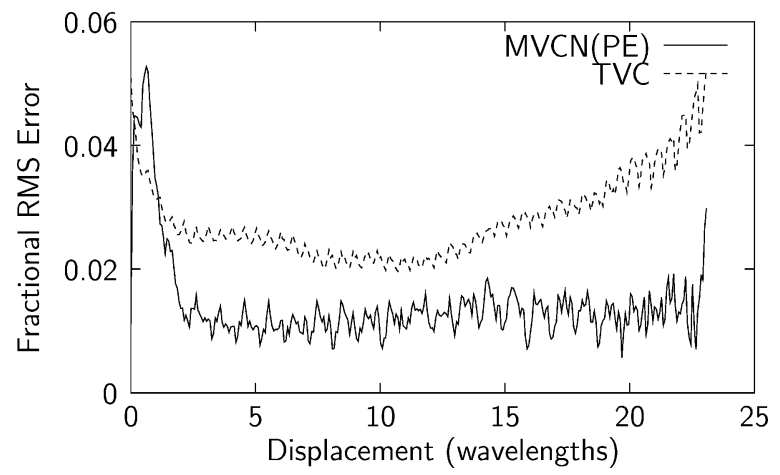

Fig. 15. RMS error in the TCD capacity metric for the TVC model and MVCN model with PE temporal correlation.

entire data window. Fig. 15 plots the fractional rms error of the TCD metric for the same set of data. In this case, the MVCN(PE) and $\mathrm{MVCN}(\mathrm{CE})$ models give nearly identical results, so only the results for $\mathrm{MVCN}(\mathrm{PE})$ are plotted.

These results suggest that the MVCN model works well for long-term channel variations. This is intuitive, since at large displacements the temporal statistics are independent and only the spatial covariance, which is properly represented at each time step, impacts the results. The failure of the model to adequately match the metrics for short displacements implies that the separable time-space assumption is rather poor. The good performance of the TVC model is somewhat remarkable, considering we only consider a small set of clusters and generate a fixed set of 50 rays for each cluster. This accuracy suggests that the random combination of a constant set of rays properly captures the short-term spatiotemporal covariance.

\section{CONCLUSION}

Although MIMO systems exhibit high capacity with perfect CSI, imperfect CSI can lead to reductions in available capacity. This paper has explored the effect of temporal variations on measured indoor and outdoor MIMO channels with receiver movement. Two metrics were developed that quantify the loss in channel quality in an information theoretic sense as either transmit or receive CSI becomes increasingly outdated. These metrics applied to indoor and outdoor $8 \times 8 \mathrm{MIMO}$ measurements at 2.55 and $5.2 \mathrm{GHz}$ indicated that although transmit CSI can be useful for tens of wavelengths, receive CSI must be updated on the order of $\lambda / 10$ for high channel capacity. This paper also introduced two modeling strategies for time-varying MIMO channels: one based on the multivariate complex normal distribution and another physical model based on an incoherent cluster modeling strategy. Both models hold promise for accurate modeling of channel temporal variation.

\section{REFERENCES}

[1] M. A. Jensen and J. W. Wallace, "A review of antennas and propagation for MIMO wireless communications," IEEE Trans. Antennas Propag., pp. 2810-2824, Nov. 2004.

[2] A. Goldsmith, S. A. Jafar, N. Jindal, and S. Vishwanath, "Capacity limits of MIMO channels," IEEE Trans. Inf. Theory, vol. 21, pp. 684-702, Jun. 2003.

[3] T. Zwick, C. Fischer, and W. Wiesbeck, "A stochastic multipath channel model including path directions for indoor environments," IEEE J. Sel. Areas Commun., vol. 20, pp. 1178-1192, Aug. 2002. 
[4] G. D. Galdo, M. Haardt, and M. Milojevic, "A subspace-based channel model for frequency selective time variant MIMO channels," in Proc. 2004 IEEE 15th Int. Symp. Personal, Indoor Mobile Radio Commun., Barcelona, Spain, Sep. 5-8, 2004, vol. 3, pp. 1603-1607.

[5] J. Maurer, C. Waldschmidt, T. Kayser, and W. Wiesbeck, "Characterisation of the time-dependent urban MIMO channel in FDD communication systems," in Proc. 2003 IEEE 57th Veh. Technol. Conf., Seoul, Korea, Apr. 22-25, 2003, vol. 4, pp. 544-548.

[6] V. Pohl, P. H. Nguyen, V. Jungnickel, and C. von Helmolt, "Continuous flat-fading MIMO channels: achievable rate and optimal length of the training and data phases," IEEE Trans. Wireless Commun., vol. 4, pp. 1889-1900, Jul. 2005.

[7] J. Maurer, "Strahlenoptisches Kanalmodell für die Fahrzeug-FahrzeugFunkkomunikation," Ph.D. dissertation, Universität Karlsruhe, , 2005.

[8] B. T. Maharaj, L. P. Linde, J. W. Wallace, and M. Jensen, "A cost-effective wideband MIMO channel sounder and initial co-located $2.4 \mathrm{GHz}$ and $5.2 \mathrm{GHz}$ measurements," in Proc. 2005 IEEE Intl. Conf. Acoust., Speech, Signal Process., Philadelphia, PA, Mar. 18-23, 2005, vol. 3, pp. $981-984$.

[9] B. T. Maharaj, J. W. Wallace, L. P. Linde, and M. A. Jensen, "Linear dependence of double-directional spatial power spectra at 2.4 and 5.2 $\mathrm{GHz}$ from indoor MIMO channel measurements," Electron. Lett., vol. 41, pp. 1338-1340, Nov. 24, 2005.

[10] B. T. Maharaj, J. W. Wallace, and M. A. Jensen, "Experimental evaluation of the MIMO wideband channel temporal variation," in Proc. 2005 URSI 28th Gen. Assembly, Delhi, India, Oct. 23-29, 2005.

[11] D. W. Bliss, K. W. Forsythe, I. Hero, A. O. , and A. F. Yegulalp, "Environmental issues for MIMO capacity," IEEE Trans. Signal Process., vol. 50, pp. 2128-2142, Sep. 2002.

[12] T. Yoo and A. Goldsmith, "Capacity of fading MIMO channels with channel estimation error," in Proc. 2004 IEEE Int. Conf. Commun., Paris, France, Jun. 20-24, 2004, vol. 2, pp. 808-813.

[13] P. Kyritsi, R. A. Valenzuela, and D. C. Cox, "Channel and capacity estimation errors," IEEE Commun. Lett., vol. 6, pp. 517-519, Dec. 2002.

[14] J. Du, Y. Li, D. Gu, A. F. Molisch, and J. Zhang, "Estimation of performance loss due to delay in channel feedback in MIMO systems," in Proc. 2004 IEEE 60th Veh. Technol. Conf., Los Angeles, CA, Sep. 26-29, 2004, pp. 1619-1622.

[15] M. Herdin, N. Czink, H. Ozcelik, and E. Bonek, "Correlation matrix distance, a meaningful measure for evaluation of non-stationary MIMO channels," in Proc. 2005 IEEE 61st Veh. Technol. Conf., Stockholm, Sweden, 30 May-1 Jun. 2005, vol. 1, pp. 136-140.

[16] G. Matz, "A time-frequency calculus for time-varying systems and nonstationary processes with applications," Ph.D. dissertation, Technische Universität Wien, , 2000.

[17] P. W. Wu and H. Lev-Ari, "Optimized estimation of moments for nonstationary signals," IEEE Trans. Signal Process., vol. 45, pp. 1210-1221, May 1997.

[18] T. Svantesson and J. W. Wallace, "Tests for assessing multivariate normality and the covariance structure of MIMO data," in Proc. 2003 IEEE Int. Conf. Acoustics, Speech, Signal Process., Hong Kong, China, Apr. 6-10, 2003, vol. 4, pp. 656-659.

[19] C. J. Mecklin and D. J. Mundfrom, "An appraisal and bibliography of tests for multivariate normality," Int. Statist. Rev., vol. 72, pp. 123-138, Apr. 2004.

[20] K. V. Mardia, J. T. Kent, and J. M. Bibby, Multivariate Analysis. New York: Academic, 1979.

[21] N. Henze and B. Zirkler, "A class of invariant consistent tests for multivariate normality," Commun. Statist. Theor. Meth., vol. 19, no. 10, pp. $3595-3618,1990$

[22] M. Steinbauer, A. F. Molisch, and E. Bonek, "The double-directional radio channel," IEEE Antennas Propag. Mag., vol. 43, pp. 51-63, Aug. 2001.
[23] Q. H. Spencer, B. D. Jeffs, M. A. Jensen, and A. L. Swindlehurst, "Modeling the statistical time and angle of arrival characteristics of an indoor multipath channel," IEEE J. Sel. Areas Commun., vol. 18, pp. 347-360, Mar. 2000.

[24] B. H. Fleury, M. Tschudin, R. Heddergott, D. Dahlhaus, and K. Ingeman Pedersen, "Channel parameter estimation in mobile radio environments using the SAGE algorithm," IEEE J. Sel. Areas Commun., vol. 17, pp. 434-450, Mar. 1999.

[25] J. Wallace, H. Özcelik, M. Herdin, E. Bonek, and M. Jensen, "A diffuse multipath spectrum estimation technique for directional channel modeling," in Proc. 2004 IEEE Int. Conf. Commun., Paris, France, Jun. 20-24, 2004, vol. 6, pp. 3183-3187.

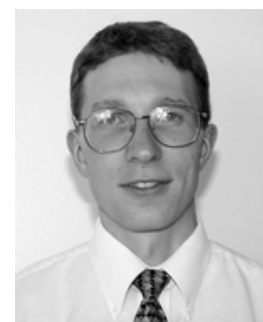

Jon W. Wallace (S'99-M'03) received the B.S (summa cum laude) and Ph.D. degrees in electrical engineering from Brigham Young University (BYU), Provo, UT, in 1997 and 2002, respectively.

From 1995 to 1997, he was an associate of Novell, Inc., Provo. During 1997, he was a Member of Technical Staff with Lucent Technologies, Denver, CO. He received the National Science Foundation Graduate Fellowship in 1998 and was a Graduate Research Assistant with BYU until 2002. From 2002 to 2003 , he was with the Mobile Communications Group, Vienna University of Technology, Vienna, Austria, pursuing collaborative research in the area of wireless channel measurement and modeling. From 2003 to 2006 , he was a Research Associate with the BYU Wireless Communications Laboratory, developing platforms for wide-band MIMO channel sounding and realtime space-time coding. He is currently an Assistant Professor at the International University of Bremen, Bremen, Germany. His research interests include wireless channel sounding and modeling, real-time MIMO implementation, optical device modeling, and remote sensing.

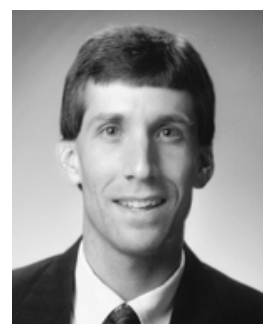

Michael A. Jensen (S'93-M'95-SM'01) received the B.S. (summa cum laude) and M.S. degrees in electrical engineering from Brigham Young University (BYU), Provo, UT, in 1990 and 1991, respectively, and the Ph.D. degree in electrical engineering from the University of California, Los Angeles (UCLA) in 1994.

From 1989 to 1991, he was a Graduate Research Assistant with the Lasers and Optics Laboratory, BYU. In 1990, he received a National Science Foundation Graduate Fellowship. From 1991 to 1994, he was a Graduate Student Researcher with the Antenna Laboratory, UCLA. Since 1994, he has been with the Electrical and Computer Engineering Department, BYU, where he is currently a Professor and Department Chair. His main research interests include antennas and propagation for personal communications, microwave circuit design, radar remote sensing, numerical electromagnetics, and optical fiber communications.

Dr. Jensen is a member of Eta Kappa Nu and Tau Beta Pi. He currently is a Member of the Administrative Committee and the Joint Meetings Committee for the IEEE Antennas and Propagation Society and an Associate Editor for the IEEE TRANSACTIONS ON ANTENNAS AND PROPAGATION. He has also served the society as Vice-Chair and Technical Program Chair for several symposia. $\mathrm{He}$ received the H. A. Wheeler Paper Award from the IEEE TRANSACTIONS ON ANTENNAS AND PROPAGATION in 2002 and the Best Student Paper Award at the 1994 IEEE International Symposium on Antennas and Propagation. 